\title{
FE-BI with UV Method for Scattering Analysis from Objects above a Lossy Half Space
}

\author{
Jian Zhu, Linlin Gong \\ Communications and Information Engineering \\ Nanjing University of Posts and Telecommunications \\ Nanjing, China
}

\author{
Mengmeng Li, Zhenhong Fan \\ Department of Communication Engineering \\ Nanjing University of Science and Technology \\ Nanjing, China
}

\begin{abstract}
In this paper, the finite-element boundary integral (FE-BI) method is applied to analyze the electromagnetic scattering from three-dimensional objects with complex media located above a lossy half space. A fast numerical approach, the UV method, is employed to decrease the memory requirement and the total CPU time for FE-BI solution. Numerical results are carried out so as to validate our developed algorithm.
\end{abstract}

Keywords-Electromagnetic scattering; finite-element boundary integral method; discrete complex image method; $U V$ method component.

\section{INTRODUCTION}

Artificial microwave complex materials, including isotropic/bi-isotropic, anisotropic/bi-anisotropic media, have been many potential applications in the fields of antennas, alteration of the radar cross sections, since the media parameter provides an extra degree of freedom to control the scattering properties of targets [1-4].In this paper, The hybrid method FE$\mathrm{BI}$ has been extended to study electromagnetic scattering from complex objects located above a lossy half space. In the boundary integral formulations, one of the most complicated issues is evaluation of the half-space dyadic Green's function [5-7]. Each component of the dyadic is represented in general via a Sommerfeld integral (SI), the direct numerical evaluation of which makes a MoM analysis prohibitive. The numerical integration of SI is time consuming since the integrand is both highly oscillating and slowly decaying. Therefore, the discrete complex image method (DCIM) [8-10] is employed to overcome this difficulty.

However, the final FE-BI system of equations consists of a partly-full, partly-sparse matrix, the equation of that is difficult to solve efficiently for iterative methods. FMM [10-13] can be extended for general targets in the presence of a lossy half space [8-9] with real-image method, which determines the large minimum group size of the FMM and leads to a low efficiency. In this paper, the rank-based methods, the multilevel UV method [14-16] is applied to overcome the above difficulty for the approximation to the half-space Green's function. The multilevel UV method is the impedance matrix decomposition based method and kernel function independent. The half-space Green's function in the "far" terms is evaluated via DCIM, and the multilevel UV method needs only to deal with the final low-ranked far matrix. Thus the approximation error is controllable via the threshold in the UV matrix compression
[14-16]. The multilevel UV method is easy to be applied in the existing FE-BI code without large change of the algorithms.

The paper is organized as follows. The formulation employed to solve the problem is reported in Section II, together with an introduction of the multilevel UV method for FE-BI analysis. In section III, numerical results are carried out so as to validate our developed algorithm. The final remarks are included in Section IV.

\section{FORMULATION}

\section{A. Weak Form of Wave Equation in complex Media and} Half-space boundary integral formulation

An arbitrary complex media object above a half space is considered. FEM is used to describe the electric field in volume $\mathrm{V}$. The wave equation has the follow equation:

$$
\left[(-\nabla+j \omega \overline{\bar{\xi}}) \bar{\mu}^{-1} q(\nabla+j \omega \overline{\bar{\zeta}})+\omega^{2} \bar{\varepsilon}\right] \mathbf{E}=-j \omega \mathbf{J}_{i}
$$

where $\bar{\varepsilon}$ and $\overline{\bar{\mu}}$ are the permittivity and permeability tensors, respectively; $\overline{\bar{\xi}}$ and $\overline{\bar{\zeta}}$ are the tensors describing the magnetoelectric coupling effects in bi-anisotropic media.

The electric and magnetic fields outside $\mathrm{S}$ can be calculated by the surface boundary integral at the surface $\mathrm{S}$. The combined-field integral equation (CFIE) is used as follows

$$
\begin{aligned}
& \hat{t}\left[\left(\mathbf{E}^{i n c}(\mathbf{r})+\mathbf{E}^{r e f}(\mathbf{r})\right)=\hat{t} \llbracket \frac{1}{2} \mathbf{E}(\mathbf{r})+j k_{0} \iint_{S} \overline{\overline{\mathbf{K}}}_{i i}^{A}\left(\mathbf{r}, \mathbf{r}^{\prime}\right)\right] \overline{\mathbf{J}}_{S}\left(\mathbf{r}^{\prime}\right) d S^{\prime} \\
& \left.\left.+\frac{j}{k_{0}} \llbracket \int_{S} \nabla \overline{\mathbf{J}}_{S}\left(\mathbf{r}^{\prime}\right) \nabla K_{i i}^{\phi e}\left(\mathbf{r}, \mathbf{r}^{\prime}\right) d S^{\prime}+\iint_{S} \nabla \times \overline{\overline{\mathbf{G}}}_{i i}^{F}\left(\mathbf{r}, \mathbf{r}^{\prime}\right)\right] \mathbf{M}_{S}\left(\mathbf{r}, \mathbf{r}^{\prime}\right) d S^{\prime}\right] \\
& \hat{n} \times\left(\overline{\mathbf{H}}^{i n c}(\mathbf{r})+\overline{\mathbf{H}}^{r e f}(\mathbf{r})\right)=\hat{n} \times\left[\frac{1}{2} \overline{\mathbf{H}}(\mathbf{r})+j k_{0} \iint_{S} \overline{\overline{\mathbf{K}}}_{i i}^{F}\left(\mathbf{r}, \mathbf{r}^{\prime}\right)\right] \mathbf{M}_{S}\left(\mathbf{r}^{\prime}\right) d S^{\prime} \\
& \left.+\frac{j}{k_{0}} \iint_{S} \nabla\left[\mathbf{M}_{S}\left(\mathbf{r}^{\prime}\right) \nabla K_{i i}^{\phi i n}\left(\mathbf{r}, \mathbf{r}^{\prime}\right) d S^{\prime}-\iint_{S} \nabla \times \overline{\overline{\mathbf{G}}}_{i i}^{A}\left(\mathbf{r}, \mathbf{r}^{\prime}\right)\right] \overline{\mathbf{J}}_{S}\left(\mathbf{r}, \mathbf{r}^{\prime}\right) d S^{\prime}\right]
\end{aligned}
$$

where $\overline{\mathbf{J}}_{S}=\hat{\eta n} \times \mathbf{H}$ and $\mathbf{M}_{S}=\mathbf{E} \times \hat{n}$ are the electric current and the equivalent magnetic current over the surface $\mathrm{S}$, respectively. $\overline{\overline{\mathbf{K}}}_{i i}^{A}, \overline{\overline{\mathbf{G}}}_{i i}^{F}, \overline{\overline{\mathbf{K}}}_{i i}^{F}, \overline{\overline{\mathbf{G}}}_{i i}^{A}$ and $K_{i i}^{\phi e}$ and $K_{i i}^{\phi m}$ are the dyadic and the scalar Green's functions for the half-space region, which can be evaluated efficiently via the complex-image technique [10]. 
RWG and Whitney basis functions are used to expand the current and electric field. Combining the formulations (1)-(3), the final FE-BI matrix equation can be obtained.

\section{B. The multilevel UV method}

The final FE-BI system of equations consists of a partlyfull, partly-sparse matrix. The dense matrix generated by BI equation is a bottleneck of the FE-BI method, which severely limits the capability of the FE-BI method in dealing with large objects. In this paper, the multilevel UV method is applied to reduce the computational complexity and memory requirement of the BI part.

The multilevel UV method is a rank-based method. Generally, the interaction matrix is full-ranked when the observation groups are in the near field of the source group, while the interaction matrix between them is low-ranked when the observation groups are in the far field. Application of the UV decomposition to the low-ranked impedance matrix will result in significant memory and computational time savings. The basic principles of the UV decomposition can be found more detail in [14]. The classification of the interaction groups in this paper is based on the octree structure [11]. The corresponding impedance matrix $\mathrm{Z}$, which represents the dense matrices in FE-BI, can be decomposed into $\mathrm{P}(\mathrm{P}$ is the number of levels) sparse matrices.

$$
\mathbf{Z}=\mathbf{Z}^{0}+\mathbf{Z}^{1}+\cdots+\mathbf{Z}^{p-1}
$$

Since $Z^{0}$ is the near impedance matrix, full-ranked and stored directly. $Z^{1}$ and so forth are the impedance matrices for the interactions of the far field. The concept of the far field used above is the same as it in the MLFMA [11].

For $\mathrm{Z}^{1}$ and so forth, the matrices are operated with two different ways according to their size, when applying the UV method. When the size of the matrix is small, it will be computed directly and its rank $r$ is evaluated by singular value decomposition (SVD), then the $\mathrm{U}$ and $\mathrm{V}$ matrixes can be obtained. When the size of the matrix is large, the column and row sampling according to rank estimates is performed and SVD on the sampled matrix is implemented instead. In the code, the rank of the first pair of groups is evaluated and the others are assumed the same as the first pair to further reduce the time for evaluation the rank. The above technique is feasible, since only the coarse estimation of the rank is needed in the UV algorithm [14]. The $m \times n$ matrix is then decomposed into $\mathrm{U}(m \times r)$ and $\mathrm{V}(r \times n)$ matrices, which leads to the significant time and memory savings when the matrix is low- ranked.

\section{NUMERICAL RESULTS}

In this section, numerical examples are considered to demonstrate the accuracy and flexibility of our FE-BI algorithm for the analysis of electromagnetic scattering from arbitrary objects in half space. All numerical experiments are performed on a Pentium 4 with $2.9 \mathrm{GHz}$ CPU and 2 GB RAM in double precision.
At first, we consider a lossy $\varepsilon_{d r}=3.0$ dielectric cylinder , that is situated $0.5 \mathrm{~m}$ above the air-soil interface. The soil is characterized by $\varepsilon_{g r}=5.0-j 0.2, \mu_{g r}=1$. A $700 \mathrm{MHz}$ plane wave is incident at $\theta_{i}=0^{0}, \varphi_{i}=0^{0}$ and the observed scattering angles are $\theta_{s}=0^{\circ} \sim 90^{\circ}$ at $\theta_{s}=0^{\circ}$. One level UV method is employed for BI part. The minimum group size is $0.236 \lambda_{0}$. As shown in Fig. 1, the numerical results of the bistatic RCS is compared with those obtained by commercial software FEKO simulation. It can be observed that there is a good agreement between them. The efficiency of the UV method for FE-BI solution is verified by comparing with the FE-BI without UV method. It takes 702.1 minutes to compute using FE-BI without UV method but only 275.8 minutes are need for the UV method. The memory requirement is $541.41 \mathrm{MB}$ for BI part without UV method, but 204.67 MB for BI part with UV method.

Fig. 2 shows the bistatic scattering cross section of a biisotropic chiral sphere in the case of $\mathrm{VV}$ - polarizations and $\mathrm{HH}$-polarizations. The chiral sphere is situated $40 \mathrm{~cm}$ above a lossy half space with a diameter of $0.6 \mathrm{~m}$. The half space is characterized by $\varepsilon_{g r}=5.0-j 0.2$ and $\sigma_{g}=0.01 \mathrm{~S} / \mathrm{m}$ at $300 \mathrm{MHz}$. Two-level UV method is employed with the minimum group size $0.076 \lambda_{0}$. The chiral body is assumed to have a relative chirality $\xi_{r}$. The chiral sphere is characterized by $\varepsilon_{r}=3.0$, $\mu_{r}=1.0$ and $\xi_{r}=0.3,0.5$. As shown in Fig. 2, the numerical results of the bistatic RCS is compared with those obtained by MoM [4]. It is obvious that an excellent agreement is obtained between them. Therefore, our algorithm has been proved to be accurate enough. The efficiency of the UV method for FE-BI solution is verified by comparing with the FE-BI without UV method. It takes 798.3 minutes to compute using FE-BI without UV method but only 265.2 minutes are need for the UV method. The memory requirement is $625.41 \mathrm{MB}$ for BI part without UV method, but 211.38 MB for BI part with UV method.

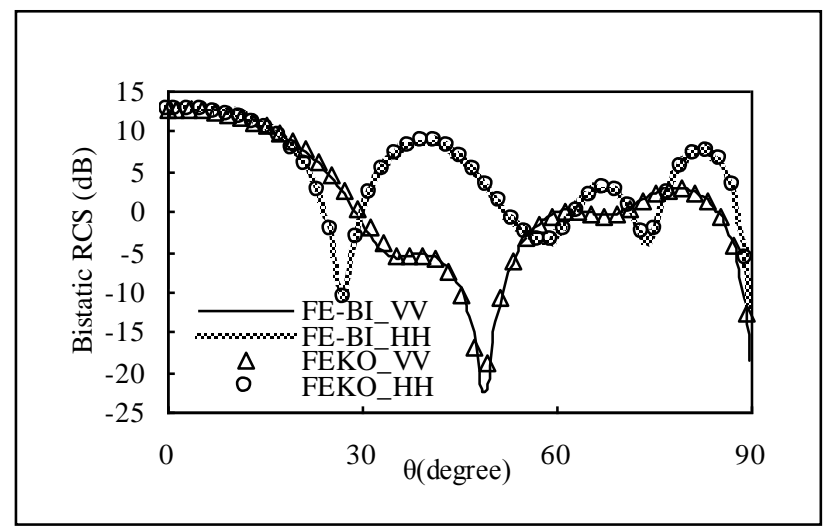

Fig. 1. Bistatic RCS from the dielectrical cylinder at $700 \mathrm{MHz}$ 


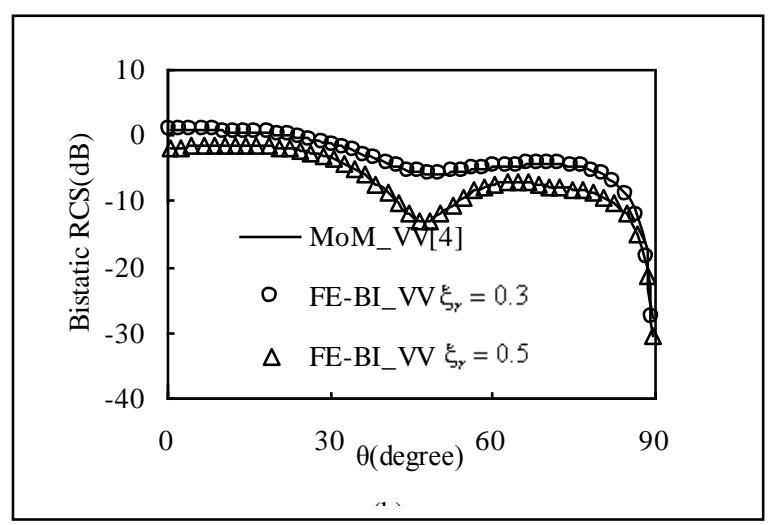

(a)

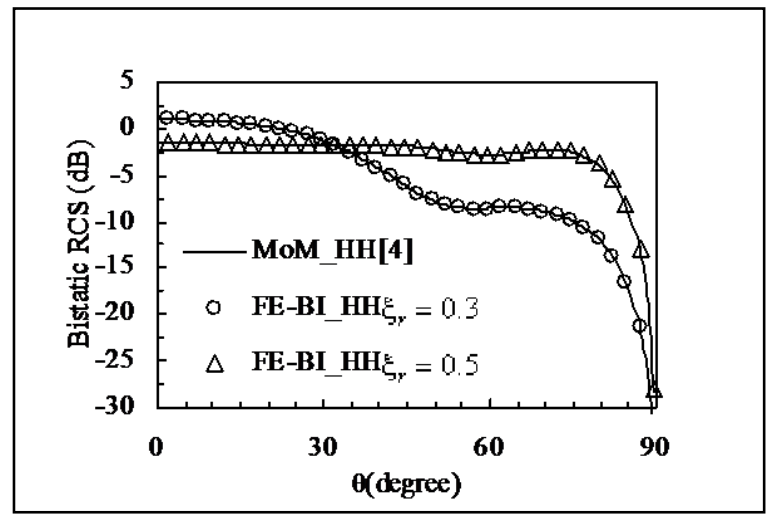

(b)

Fig. 2. Bistatic scattering cross section of a homogeneous chiral sphere above a lossy half space (a)VV-polarization and (b) HH-polarization

\section{CONCLUSIONS}

In this paper, the FE-BI method has been developed for the analysis of the electromagnetic scattering from objects with complex martial located above a lossy half space. The accuracy of the proposed method has been proved. The multilevel UV method has been successfully applied to FE-BI to decrease the memory requirement and CPU time of solution.

\section{ACKNOWLEDGMENT (HEADING 5)}

We would like to thank the support of Jiangsu Natural Science Foundation of BK2011757, Scientific Research Foundation of Nanjing University of Posts and Telecommunications of NY210071 and NY212021.

\section{REFERENCES}

[1] Volakis J. L., A. Chatterjee, and L. C. Kempel, Finite Element Method for Electromagnetics: Antennas, Microwave Circuits and Scattering Applications, IEEE Press, New York, 1998.
[2] Fu-Gang Hu, Chao-Fu Wang, and Yeow-Beng Gan. "Efficient Calculation of Electromagnetic Scattering from Cavities Coated with Bianisotropic Media Using FE-BI Method with HigherOrder Tetrahedral Elements," IEEE Antennas and Propagation Society International Symposium, pp. 3883-3886, July 2006.

[3] Filiberto Bilotti, Lucio Vegni and Alessandro Toscano, "Radiation and Scattering Features of Patch Antennas With Bianisotropic Substrates," IEEE Transactions On Antennas And Propagation, vol. 51, no. 3, pp. 449-456, March 2003.

[4] X. D. Wang, Douglas H. Werner, Le-Wei Li, Yeow-Beng Gan. "Interaction of Electromagnetic Waves With 3-D Arbitrarily Shaped Homogeneous Chiral Targets in the Presence of a Lossy Half Space," IEEE Trans. Antennas Propag., vol. 55, no. 12, Dec 2007.

[5] X. Yuan, D. R. Lynch, and J.W. Strohbehn, "Coupling of finite element and moment methods for electromagnetic scattering from inhomogeneous objects," IEEE Trans. Antennas Propagat., vol.38, pp.386-393, Mar. 1990.

[6] Y. Zhang, X. Wei, and E. Li, "Electromagnetic Scattering from three-dimensional Bianisotropic Objects Using Hybrid Finite Element-Boundary Integral Method," J. of Electromagn. Waves and Appl., Vol. 18, No. 11, 1549-1563, 2004.

[7] J. Zhu, M.M. Li, Z.H. Fan, and R.S. Chen, "Analysis of EM Scattering from 3D Bi-anisotropic Objects above a Lossy Half Space Using FE-BI with UV Method," unpublished.

[8] R. S. Chen, Y. Q. Hu, Z. H. Fan, D. Z. Ding, D. X. Wang and E. K. N. Yung, "An Efficient Surface Integral Equation Solution to EM Scattering by Chiral Objects above a Lossy Half space", IEEE Trans. Antennas Propagt., vol. 57, no. 11, pp. 3586-3593, Nov. 2009.

[9] Giuseppe Pelosi, Giovanni Toso, and Roberto Coccioli, "Finiteelement boundry-integral analysis of electromagnetic scattering by a buried dielectric object," Microwave And Optical Technology Letters, vol. 24, no. 6, March 20, 2000.

[10] D. G. Fang, J. J. Yang, and G. Y. Delisle, "Discrete image theory for horizontal electric dipole in a multilayer medium," Proc. Inst. Elect. Eng. H, vol. 135, no. 5, pp. 297-303, Oct. 1988

[11] W. C. Chew, J. M. Jin, E. Midielssen, and J. M. Song, Fast and Efficient Algorithms in Computational Electromagnetics, Boston, MA: Artech House 2001.

[12] Z. Liu, J. He, Y. Xie, A. Sullivan and L.Carin, "Multilevel Fast Multipole Algorithm for General Targets on a Half-Space Interface," IEEE Trans. Antennas Propag., Vol. 50, No. 12, pp.1838-1849, Dec. 2002.

[13] L. B. Felsen and N. Marcuvitz, Radiation and Scattering of Waves.Piscataway, NJ: IEEE Press ch. 4, 1996.

[14] L. Tsang, Q. Li, P. Xu, D. Chen, and V. Jandhyala, "Wave scattering with UV multilevel partitioning method: 2. Threedimensional problem of nonpenetrable surface scattering," Radio Sci., vol. 39, p. RS5011,2004.

[15] C. J. Ong and L. Tsang, "Full-wave analysis of large-scale interconnects using the multilevel UV method with the sparse matrix iterative approach (SMIA)," IEEE Trans. Antennas Propag., Vol. 31, No. 4, pp.818-829, Nov. 2008.

[16] K. L. Lai, L. Tsang and C. -J. Ong, "Application of multilevel UV method to analyze large microstrip patch structures," IEEE Antennas and wireless propagation letters, Vol. 4, pp.471-474, 2005. 